\title{
Eficacia de un Programa de Innovación Docente en el Rendimiento, la Adquisición de Competencias Específicas y la Autoevaluación del Alumnado
}

\section{Effectiveness of a Teaching Innovation Program in the Academic Performance, the Acquisition of Specific Competences and the Self-Assessment of the Students}

\author{
Mª del Mar Benítez Hernández *, Juan Francisco Rodríguez-Testal y Macarena Torrado Herrera \\ Universidad de Sevilla, España
}

\section{DESCRIPTORES:}

Innovación pedagógica

Rendimiento académico

Competencias

Autoevaluación

Adaptaciones

curriculares

\section{RESUMEN:}

En el contexto académico actual el aprendizaje por competencias implica la renovación a metodologías más activas que se acerquen a la realidad profesional, y cambios en la forma de evaluar. El presente estudio, realizado durante los cursos 2017/2018 y 2018/2019, trata de valorar la eficacia de una experiencia de innovación docente en la adquisición de competencias específicas, el rendimiento académico y la autoevaluación de 44 estudiantes del Máster de Psicología General Sanitaria (MPGS). En concreto, el alumnado participó en el diseño y desarrollo de un programa de entrenamiento de habilidades sociales (HHSS) para pacientes, actuando como terapeuta-coterapeuta (participación directa), y/u observador-registrador (participación indirecta). Asimismo, se incorporó a la experiencia a dos alumnas invidentes para determinar qué tipo de adaptaciones se podrían llevar a cabo. Los resultados muestran que el alumnado ha adquirido las competencias específicas evaluadas y se ha producido una mejora del rendimiento académico, así como de la autoevaluación global y por competencias. A este respecto, no se encontraron diferencias entre el alumnado en función de su participación. Por otro lado, se hallaron diferencias entre la evaluación y autoevaluación, aunque con un tamaño del efecto bajo al concluir el programa. Finalmente, se discuten los hallazgos y las adaptaciones curriculares.

\section{ABSTRACT:}

In the current academic context learning by competencies implies the renewal of more active methodologies that approach the professional reality, and changes in the way of assessing. The present study, carried out during the 2017/2018 and 2018/2019 school years, tries to evaluate the effect of a teaching innovation experience in the acquisition of specific competences, academic performance, and self-assessment of 44 students of the Master's Degree in General Health Psychology. Specifically, the students participated in a design and development of social skills training program for patients in two way, as therapist-cotherapist (direct participation) and/or observer-recorder (indirect participation). Also, two blind students were incorporated into the experience to assess what kind of adaptations could be made. The results show that students have acquired the specific competences evaluated and the academic performance has improved as well as global self-assessment and by competencies have improved too. On this matter, based on participation, no differences were found among students. On the other hand, based on evaluation, differences were found according to evaluation or self-assessment, although with a smaller size effect at the end of the program. Finally, results and curricular adaptations are discussed.

CÓMO CITAR:

Benítez-Hernández, M. M., Rodríguez Testal, J. F. y Torrado, M. (2021). Eficacia de un programa de innovación docente en el rendimiento, la adquisición de competencias específicas y la autoevaluación del alumnado. Revista Iberoamericana de Evaluación Educativa, 14(1), $191-208$. https://doi.org/10.15366/riee2021.14.1.011

*Contacto: marbenitez@us.es ISSN: 1989-0397

https://revistas.uam.es/riee 


\section{Introducción}

Los retos planteados por el Espacio Europeo de Educación Superior (en adelante EEES) suponen un cambio en el paradigma educativo tanto en la naturaleza del aprendizaje como en el papel de la evaluación. Actualmente, la educación universitaria española está inmersa en el proceso de adaptación que supone la formación por competencias, la renovación a metodologías más activas que se acerquen a la realidad profesional y la evaluación como estrategia positiva de aprendizaje (Castañeda, 2016; Fernández, 2010; Tejada y Ruiz, 2016; Zabalza, 2013).

Envueltos en este proceso de cambio, no podemos olvidar que la innovación docente debe estar debidamente justificada, ser viable y práctica y estar pensada desde la mejora y actualización de la formación. Además, debe ser documentada y evaluada, contrastando su eficacia, eficiencia y efectividad. En palabras de Zabalza (2013), "innovar no es solo hacer cosas distintas sino hacer cosas mejores" (p.117).

Por ello, antes que nada, nos gustaría delimitar el concepto de aprendizaje por competencias, puesto que ocupa un lugar central en la formación superior derivada del EEES. Aunque no existe una definición consensuada del término, la Recomendación del Parlamento Europeo y del Consejo relativa a la creación del Marco Europeo de Cualificaciones para el Aprendizaje Permanente la define como:

Demostrada capacidad para utilizar conocimientos, destrezas y habilidades personales, sociales y/o metodológicas, en situaciones de estudio o de trabajo y en el desarrollo profesional y/o personal. En el Marco Europeo de Cualificaciones, la competencia se describe en términos de responsabilidad y autonomía. (UE, 2008, pp. C111/4)

Profundizando un poco más, encontramos que otros autores describen las competencias como un conjunto de conocimientos, capacidades y aptitudes que se ponen en práctica para resolver una situación o problemática determinada, resaltando que son adquiridos mediante el aprendizaje y la experiencia (Castañeda, 2016; Fernández, 2010; Rodríguez, 2007; Tejada y Ruiz, 2016).

Por lo tanto, con relación al aprendizaje por competencias, podemos afirmar que ya no se pone el énfasis en los contenidos sino en el aprendizaje orientado a la solución de problemas, otorgando un papel activo y reflexivo al alumnado, que fomente el aprendizaje autónomo pero guiado de los estudiantes (Fernández, 2010; Zabalza, 2013) y adaptando la formación universitaria a las exigencias del mundo laboral (Rodríguez, 2007). Es decir, dotando al aprendizaje de funcionalidad con la intención de que se alcancen las competencias profesionales de forma práctica y motivada (Valle y Manso, 2018). Existe evidencia científica de que el alumnado que aprende de manera activa aplica mejor los conocimientos adquiridos y se encuentran más satisfechos con el proceso de aprendizaje (Armbruster et al., 2009; Ramos-Villagrasa et al., 2019).

En este sentido, muchos autores relacionan al rendimiento académico con el logro de un complejo proceso de aprendizajes que implican a la autoeficacia, autorregulación y ajuste emocional. Se trata de buscar una mayor implicación y autonomía del alumnado, la integración de sus potencialidades, el uso adaptativo de sus estrategias, estilos de afrontamiento, y regular la percepción de que las calificaciones son una consecuencia derivada de estos procesos de aprendizaje (Rodríguez et al., 2011; Tipismana, 2019).

Del mismo modo, esta metodología de enseñanza-aprendizaje puede beneficiar las adaptaciones curriculares. Las personas con discapacidad se encuentran con muchas de las barreras del sistema universitario descritas anteriormente, como un enfoque de aprendizaje por contenidos, metodologías poco dinámicas, etc. Con respecto a esto, los estudios señalan que una metodología didáctica basada en la participación beneficia la formación de este colectivo y consideran que, asimismo, un diseño de programa inclusivo de buenas prácticas de enseñanza revertirá en la mejora del aprendizaje de todo el alumnado (Adams y Holland, 2006; Moriña-Diez et al., 2013).

\section{Revisión de la literatura}

Como podemos observar son muchos los cambios, modificaciones y adaptaciones que pueden ser llevadas a cabo para cumplir con los objetivos propuestos por el EEES.

En una investigación realizada por Castañeda (2016) se planteó si los Planes de Estudio están realmente desarrollando los principios fundamentales de la formación por competencias. Para ello, evaluaron 
mediante entrevistas, cuestionarios y grupos de discusión, a docentes y alumnos/as. Los resultados han puesto de manifiesto que la formación universitaria aún dista de alcanzar una educación basada en competencias. Por ejemplo, entre otros aspectos, se observó que falta una mayor integración de los perfiles profesionales durante el proceso de enseñanza-aprendizaje, que aún se mantienen metodologías más teóricas que prácticas, que habría que delimitar más las competencias profesionales y mejorar el sistema de evaluación del aprendizaje.

Con relación a la profesión del psicólogo/a existe gran interés en conocer cuáles son las competencias que se deben adquirir, así como la manera de evaluar esa adquisición. La American Psychological Association (APA) es la que ha ofrecido la definición más completa sobre las competencias de los profesionales de la psicología, influyendo en programas formativos a nivel mundial (Lasa, 2007). Asimismo, destacan las publicaciones llevadas a cabo por la Conferencia de Competencias desde 2007. Por un lado, los trabajos de Hatcher y Lassiter (2007) donde concretan las competencias de carácter práctico que debe adquirir el psicólogo. Y por el otro, el de Fouad y otros (2009) que señala los criterios que se deben considerar en cada competencia y su forma de evaluar.

Actualmente en España los programas docentes de los Másteres de Psicología General Sanitaria (MPGS) se centran en garantizar las competencias establecidas en la orden ECD/1070/2013 por la que se establecen los requisitos para la verificación de los títulos universitarios oficiales de MPGS que habilite para el ejercicio de la profesión titulada y regulada de Psicólogo/a General Sanitario.

En un estudio realizado por Jenaro y otros (2016) se evaluó la adquisición de competencias establecidas en el MPGS de la Universidad de Salamanca a través de una encuesta a 244 estudiantes. Los resultados mostraron que el alumnado presentaba elevada satisfacción con la adquisición de competencias teóricas y prácticas a través del prácticum, siendo las valoraciones medias-bajas en las asignaturas teóricas-practicas. Estos hallazgos están en coherencia con lo argumentado anteriormente, demostrando la necesidad de mejorar las metodologías didácticas empleadas en las asignaturas teórico-prácticas. Asimismo, los estudiantes realizaban una autoevaluación final en cada asignatura donde valoraban su aprendizaje con relación a dichas competencias específicas. En la literatura hemos encontrado que muchos estudios defienden la incorporación de este tipo de evaluación en la universidad.

Por un lado, destaca el trabajo de Boud (1995) que define la autoevaluación como la participación activa y responsable del alumnado en la realización de juicios sobre su propio aprendizaje. Este autor ha llevado a cabo numerosos estudios y en su revisión de los procesos de autoevaluación y su relación con el aprendizaje, no encontró diferencias significativas entre las puntuaciones del alumnado y del profesorado al utilizar este tipo de técnicas (como se citó en Fernández, 2010).

Por su parte, Dochy et al. (1999) concluyeron que la autoevaluación es un proceso que mejora con el tiempo y especialmente con la retroalimentación. A este respecto, diversos autores coinciden en que la capacidad de valorar el propio progreso ya es parte de las competencias (Fernández, 2010; Gimeno y Gallego, 2007). En este sentido, Bronkbank y MacGill (2002), exponen que la autoevaluación promueve el aprendizaje reflexivo. Asimismo, Villardón (2006) señala que proporciona estrategias de desarrollo personal y profesional como la capacidad crítica, la autonomía, el compromiso, la responsabilidad y la motivación para el aprendizaje.

Al respecto, la forma de ser y ejercer la labor docente influye en la construcción de la identidad profesional de los y las estudiantes (Colomo y Gabarda, 2019). Aunque cada estudiante responde a un estilo particular y predominante de aprendizaje, según Saul y otros (2016), si es estimulado por estrategias de enseñanza adecuadas se fomentará la construcción de su propio conocimiento y con ello la mejora de su rendimiento. Por ello, es necesario que la labor del docente no solo se limite a la transmisión de información y conocimientos, sino que se trata de que puedan plantear situaciones al alumnado que se acerquen a la realidad profesional, donde manifestándose alguna dificultad, los alumnos y alumnas pueda reflexionar y demostrar cómo hacen uso de sus conocimientos, habilidades y actitudes para establecer respuestas a las problemáticas que se planteen (Delors, 1997; Villardón, 2006). Del mismo modo, debemos tener en cuenta que la evaluación del aprendizaje por competencias implica mayor complejidad, puesto que hay que contemplar además de la adquisición de los conocimientos, las habilidades y actitudes (Castañeda, 2016; Tejada y Ruiz, 2016). Asimismo, pensando en lograr un aula inclusiva es esencial crear un clima de comunidad donde el profesorado y los estudiantes trabajen conjuntamente. El trabajo en grupo tiene un 
gran potencial para crear entornos educativos más ricos (Castellana y Sala, 2005), y cuando se establece una interacción más dinámica, la retroalimentación permite aprender de la experiencia (Abejón Mendoza et al., 2010).

En un estudio realizado por García Cerdeño y otros (2016) con estudiantes con discapacidad visual de la escuela de Psicología Clínica de la Universidad Técnica de Manabí se proponen algunas acciones que contribuyeron a una mejor inclusión y que coinciden con otros autores (Abejón Mendoza et al., 2010; Gross, 2014). Estas van orientadas a una mayor sensibilización de los compañeros, docentes y personal universitario, así como a la capacitación de los/las profesores en este ámbito. Entre ellas, destacamos que los alumnos con discapacidad no deben ser contemplados como sujetos pasivos sino como protagonistas de su propio proyecto educativo y programa de integración, y que es importante tener en cuenta un reajuste de cualquiera de los elementos curriculares para dar respuesta a las condiciones particulares de cada alumno/a con discapacidad sensorial visual. Al respecto, se han detectado como dificultades más significativas en este colectivo la falta de acceso a ayudas técnicas y/o económicas, necesidad de adaptación de materiales, exámenes o pruebas, problemas con metodologías visuales como el uso de ordenadores, diapositivas o pizarra, entre otras (Abejón Mendoza et al., 2010). Por otro lado, la figura del compañero de clase es un recurso muy valorado (Castellana y Sala, 2005). También la ubicación del estudiante dentro del aula, el uso de recursos verbales, la adaptación de materiales y el aumento de tiempos (Abejón Mendoza et al., 2010. Gross, 2014).

Conforme a lo expuesto podemos decir que, en la formación superior en general, y de los psicólogos y las psicólogas en particular, aún se estima necesario continuar trabajando en la mejora de metodologías didácticas, adaptaciones curriculares y procesos de evaluación con el objetivo de que los futuros profesionales salgan de la universidad mejor capacitados para realizar su labor. Con relación a esto, llevar a la práctica los contenidos aprendidos, así como hacer adaptaciones e incorporar mejoras o nuevas formas de evaluación, es fundamental para desarrollar las competencias que de estos profesionales se esperan y demandan.

En línea de lo anterior, Rodríguez-Testal y otros (2009) observaron que el análisis de historias clínicas, o el desempeño de papeles según un guion de prototipo clínico entre alumnos/as de psicología de la Universidad de Sevilla eran insuficientes para la formación del alumnado. Desde el curso académico 1995-1996, estos autores tratando de acercar más el contexto clínico a las aulas, decidieron incorporar a su método de enseñanza la visualización de videos, al principio editados por otros autores y luego de casuística propia, que contenían entrevistas clínicas y seguimiento de pacientes. Posteriormente, continuando con la idea de acercar lo más posible la realidad a la que se enfrenta el psicólogo en las sesiones, Rodríguez-Testal y León-Fuentes (2002) propusieron que fueran los alumnos/as quienes, con supervisión, elaboraran el material para las entrevistas, participaran durante la grabación y expusieran en clase la experiencia. De esta manera, la implicación personal y el trabajo activo de los estudiantes conlleva una participación activa de los mismos en la construcción de su propio aprendizaje (De Miguel, 2006), consiguiendo así un sentimiento de autonomía que puede funcionar como estrategia de motivación para los alumnos (Álvarez et al., 2007). Además, con este material crearon una base de casos clínicos de uso para alumnos/as y docentes, no limitándose al uso exclusivo en el aula (Rodríguez-Testal et al., 2017).

En trabajos previos, Benítez-Hernández y otros (2019) demostraron una mejora del rendimiento académico global del alumnado a través de su participación en sesiones con pacientes con trastorno psicológico y déficit en habilidades sociales, contrastando que al final del curso, todas las competencias relacionadas con la intervención con pacientes fueron alcanzadas por el alumnado, así como observaron una disminución de la sintomatología ansioso-depresiva y un aumento de las conductas de interacción social de los pacientes. Además de los beneficios que el alumnado puede obtener en su formación al participar en un contexto real de intervención terapéutica, al mismo tiempo este tipo de programas puede presentar beneficios saludables para los pacientes, a los que se les proporciona herramientas a través de técnicas de entrenamiento en asertividad, y solución de problemas y ensayo de conducta, siguiendo un modelo de Terapia Dialéctico Conductual (TDC) (Linehan, 2003). Con la TDC el paciente mejora en autoestima y confianza en sí mismo, cuando empatiza con su comportamiento, pensamiento, y emociones, los valida, y acepta que éstos tienen sentido al considerar su historia y las circunstancias en las que vive (de Flores et al., 2007; García-Palacios, 2006; Gómez, 2007). 
En definitiva, a partir de los objetivos marcados por el EEES y a la luz de las teorías y hallazgos expuestos, consideramos que aun debemos mejorar las metodologías participativas y procesos de evaluación por competencias, así como evaluar los resultados alcanzados con estas propuestas.

En este contexto surge la iniciativa del presente trabajo, en el que se pretende valorar un programa de innovación docente que da continuidad a los expuestos anteriormente, y que ha sido propuesto por los docentes de la asignatura "Salud en alteraciones mentales: propuesta para la intervención psicológica" del MPGS de la Universidad de Sevilla. Con el objetivo de que los alumnos y alumnas puedan adquirir y/o mejorar las competencias incluidas en el programa de dicha asignatura y que coinciden con las establecidas en la orden ECD/1070/2013, los profesores ofrecen al alumnado la oportunidad de poner en práctica sus conocimientos, habilidades y aptitudes a través de la participación en sesiones supervisadas de entrenamiento de habilidades sociales (HHSS) para pacientes con trastorno psicológico y déficit en habilidades sociales. Las sesiones se llevan a cabo en un aula de la Facultad de Psicología de Sevilla, acondicionada con una sala con espejo unidireccional y sistema de grabación. El alumnado puede participar de forma directa (PD) como terapeuta-coterapeuta de una de las sesiones y también de forma indirecta (PI) como observador-registrador a partir del visionado de éstas. Asimismo, pueden tener la ocasión de formar parte del equipo reflexivo en una o varias sesiones.

Por tanto, tratando de superar limitaciones de investigaciones previas (Benítez-Hernández et al., 2019), en el presente estudio nuestro objetivo fue evaluar la eficacia de la intervención psicológica realizada por el alumnado de la asignatura "Salud en alteraciones mentales: propuesta para la intervención psicológica” del MPGS de la Universidad de Sevilla (cursos 2017/2018 y 2018/2019) en el rendimiento académico global, la adquisición de competencias específicas y la autoevaluación del alumnado. Además, se tendrá en cuenta si se han beneficiado más, en estos términos, los estudiantes que participan directamente en las sesiones como terapeuta-coterapeuta frente a los que solo participan como observadores-registradores. También se evaluará si existen diferencias en función de la evaluación (rendimiento académico global) y la autoevaluación global. Asimismo, incorporamos a la experiencia a dos alumnas invidentes para valorar qué tipo de adaptaciones se podrían llevar a cabo.

\section{Método}

\subsection{Participantes}

En este estudio participaron voluntariamente un total de 44 alumnos y alumnas del Máster de Psicología General Sanitaria de la Facultad de Psicología de Sevilla. En concreto, de la asignatura "Salud en alteraciones mentales: propuesta para la intervención psicológica" de los cursos 2017/2018 y 2018/2019. La muestra se obtuvo mediante muestreo no probabilístico por accesibilidad, con todos los participantes de la asignatura en la que se llevó a cabo la innovación docente.

Del total de participantes, 9 eran hombres y 35 mujeres, con edades comprendidas entre 22 y 45 años y un nivel socioeconómico medio. Asimismo, 29 de estos alumnos/as realizaron una participación directa (PD) como terapeutas-coterapeutas en el programa de entrenamiento de HHSS para pacientes con trastorno psicológico y déficit en habilidades sociales, es decir, prepararon y llevaron a cabo una de las seis sesiones de su respectivo curso con la supervisión de los docentes. Por otro lado, los 15 alumnos/as restantes, participaron de forma indirecta (PI), a través del visionado de las sesiones, como observadores-registradores, llevando a cabo el seguimiento de uno de los pacientes participantes y con la opción de participar en el equipo reflexivo. El alumnado de participación directa se unió al grupo anterior en el resto de las sesiones que no actuó como terapeuta-coterapeuta, asumiendo el papel de observadores-registradores. También se incorporó a la experiencia a dos alumnas invidentes para determinar qué tipo de adaptaciones se podrían llevar a cabo.

Por último, hay que mencionar que de la muestra total inicial (48 alumnos/as) solo tuvimos en cuenta aquellos estudiantes que participaron en el cuestionario de evaluación inicial y final (44 alumnos/as), siendo eliminados del estudio 4 estudiantes que no cumplían con este criterio. Además, con relación a la prueba de autoevaluación, 40 alumnos/as cumplimentaron el cuestionario, teniendo para esta variable una pequeña pérdida muestral (4 alumnos/as). 


\subsection{Variables de estudio}

Autoevaluación. Valoración del alumnado respecto a la asignatura y a su ejercicio profesional, de forma global y por competencias (conocimientos, habilidades o técnicas y responsabilidad) desarrolladas durante las sesiones de entrenamiento de HHSS. Se miden a través de las puntuaciones obtenidas en la pregunta 9.3 del cuestionario de evaluación inicial y final elaborado por el profesorado.

Competencias específicas (CE). Adquiridas por el alumnado a través de la participación (directa y/o indirecta) en el programa de entrenamiento de HHSS. Se miden a través de las puntuaciones obtenidas en determinadas preguntas del cuestionario de evaluación inicial y final elaborado por los profesores. Para ello, se utilizó un documento elaborado por los autores de esta experiencia, donde se puede consultar las 13 competencias específicas, y las preguntas y puntuaciones del cuestionario que evalúan su adquisición.

Participación directa o indirecta. En la participación directa (PD) como terapeuta-coterapeuta en el programa de entrenamiento de HHSS, el alumnado diseñó y llevó a cabo una de las seis sesiones de su respectivo curso con la supervisión de los docentes. En la participación indirecta (PI) el alumnado actuó como observador-registrador a través del visionado de las sesiones, llevando a cabo el seguimiento de uno de los pacientes participantes.

Programa de entrenamiento de HHSS para pacientes con trastorno psicológico y déficit en habilidades sociales. Intervención psicológica grupal de seis sesiones, diseñadas y llevadas a cabo por parte del alumnado que participaba como terapeuta-coterapeuta, y visionadas por el resto, todo bajo la supervisión del profesorado. Se emplearon técnicas de entrenamiento en asertividad, solución de problemas y ensayo de conducta, siguiendo un modelo de TDC (Linehan, 2003).

Rendimiento académico. Adquiridos por el alumnado durante el desarrollo de la asignatura, y evaluado por el profesorado a través de una prueba, que ellos mismos habían elaborado previamente. Se mide a partir de la puntuación global obtenida en el cuestionario de evaluación inicial y final.

\subsection{Instrumentos}

\subsubsection{Cuestionario de evaluación inicial y final}

Esta prueba elaborada por el profesorado de la asignatura consta de 9 preguntas abiertas, de las cuales 6 están a su vez compuestas por 2 o más preguntas.

Este cuestionario no fue sometido a un proceso de evaluación estricto. Como se indica en el punto siguiente, se fundamentó en poder responder y evaluar diferentes competencias. Se considera que el cambio registrado de la primera evaluación a la segunda en los diferentes aspectos que lo integran, son evidencias de validez ecológica. No se realizó una evaluación interjueces de cada medida en los dos momentos de evaluación como criterio de fiabilidad, pero se establecieron las directrices que debían cumplirse en las distintas respuestas para su puntuación por el mismo evaluador.

Respecto a la evaluación del rendimiento global del alumnado, se ha tenido en cuenta las puntuaciones obtenidas en las preguntas de la 1 a la 9.2, considerando mayor el rendimiento a medida que aumenta la puntuación. Por lo tanto, en función de la puntuación obtenida (en un rango de 0 a 10), se clasificó la puntuación en cuatro niveles: de 0 a 4,9 "suspenso", de 5 a 6,9 "aprobado", de 7 a 8,9 "notable" y de 9 a 10 "sobresaliente".

Asimismo, para valorar la adquisición de las competencias específicas, se tuvo en cuenta la relación de CE con las preguntas de este cuestionario que las evalúan y las puntuaciones para considerarlas superadas.

Por otra parte, el cuestionario incluye la autoevaluación del alumnado (pregunta 9.3) que consta de 4 ítems, en una escala tipo Likert, con valores de 0 a 5, que posteriormente fueron ponderados en un rango de 0 a 10. En función de la puntuación obtenida, las preguntas sobre los conocimientos, habilidades/ técnicas y responsabilidad (9.3.1, 9.3.2, y 9.3.3, respectivamente) se clasificaron siguiendo el criterio de: 0 a 4.9 "no superada" y de 5 a 10 "superada". Por otro lado, la valoración global se clasificó de la siguiente forma: de 0 a 4,9 “suspenso", de 5 a 6,9 "aprobado", de 7 a 8,9 "notable" y de 9 a 10 "sobresaliente". 


\subsubsection{Relación de competencias específicas, adquiridas a través de la experiencia de innovación docente, preguntas que las evalúan y puntuaciones para considerarlas superadas}

Elaboramos un documento donde se recoge la relación de preguntas que evalúan cada una de las 13 competencias específicas que se han trabajado a través de esta metodología de innovación didáctica. Se estableció que la competencia específica era alcanzada si la suma de las puntuaciones que la forman supera el $50 \%$ de su valor.

Por último, resaltar que las CE contempladas fueron extraídas del programa de la asignatura, que previamente fue consensuado y aprobado por el profesorado, la Junta y el Rector, y que además corresponden con las competencias establecidas en la orden ECD/1070/2013.

\subsubsection{Valoración de las dos alumnas invidentes sobre la experiencia del grupo de terapia}

Documento no estructurado que recoge la valoración de cada alumna sobre las adaptaciones curriculares que se habían llevado a cabo, su vivencia y sugerencias o propuestas de mejora.

\subsection{Fases del estudio}

Las fases del presente estudio son tres: evaluación inicial, diseño e implementación del entrenamiento de HHSS para pacientes con trastorno psicológico y déficit en habilidades sociales y evaluación final.

Evaluación inicial. En la presentación de la asignatura, los profesores ofrecieron al alumnado, como metodología innovadora, la propuesta de mejorar en sus conocimientos, habilidades y aptitudes mediante un entrenamiento de HHSS destinado a ocho pacientes voluntarios. Estos pacientes fueron previamente seleccionados por los docentes en base a dos criterios: que tuvieran un diagnóstico de trastorno psicológico y, además, un déficit en HHSS.

Tras la aceptación de la propuesta por parte del alumnado, estos realizaron la firma del consentimiento informado.

Posteriormente se ofreció a todos los alumnos y alumnas la posibilidad de ser terapeutas-coterapeutas (PD). Aquellos que se presentaron voluntarios recibieron al azar una de las seis sesiones propuestas por cada curso académico.

Asimismo, los profesores explicaron la forma de registrar conductas de interacción social mediante la observación de los pacientes para que, posteriormente, el alumnado visionando las sesiones como observador-registrador (PI), pudiera realizar el seguimiento. A cada alumno/a se le asignó aleatoriamente un/a paciente.

Por último, se administró al alumnado el cuestionario de evaluación elaborado por los docentes.

Con relación a las dos alumnas invidentes, los docentes se reunieron con ellas para valorar y acordar qué adaptaciones podrían llevarse a cabo para sus participaciones en la experiencia del grupo de terapia.

Implementación de la metodología docente innovadora: entrenamiento de habilidades sociales para pacientes con trastorno psicológico y déficit en habilidades sociales. De manera paralela al desarrollo de las clases teórico-prácticas, el profesorado supervisaba las propuestas de intervención planteadas por el alumnado antes de cada sesión, orientándolos y resolviendo dudas. Las clases donde se llevaban a cabo las sesiones se realizaban los jueves, en horario de 18:30h a 21:00h en el aula de observación, dotada de un espejo unidireccional y equipo técnico de grabación, y ubicada en la misma Facultad de Psicología de la Universidad de Sevilla.

En total se llevaron a cabo seis sesiones en cada curso (2017/2018 y 2018/2019). Cada sesión fue grabada en vídeo para su uso en revisiones y como material educativo. El contenido desarrollado estuvo basado en técnicas de entrenamiento en asertividad, solución de problemas, y ensayo de conducta, siguiendo un modelo de TDC (Linehan, 2003).

Estas clases, donde se incluía el desarrollo de las sesiones, seguían la siguiente estructura:

- 18:30h a 19:00h. En el aula se explicaba que se iba a hacer en la sesión, los temas que se iban a tratar, si se había quedado pendiente algo en la sesión anterior para trabajarlo en esta y las tareas para casa que se mandarían al final. 
- 19:00h a 20:30h. Puesta en práctica, con supervisión, de la intervención diseñada por los alumnos/as (terapeuta-coterapeuta). El resto del alumnado (observadores-registradores), realizaba el visionado de la sesión y el seguimiento de los/as pacientes a través del registro de conductas. El desarrollo general de la sesión consistía en: presentación del terapeuta y coterapeuta al grupo, preguntas sobre las tareas de la semana previa, realización de las dinámicas, establecimientos de las próximas tareas y cierre de la sesión. No obstante, en el transcurso de las sesiones se realizaban los ajustes necesarios en función de las problemáticas que surgían. Además, nos gustaría resaltar que en todas las sesiones uno de los docentes acompañaba dentro de la sala a los/as terapeuta-coterapeuta, mientras que el otro se quedaba acompañando al resto de los alumnos/as que se encontraban en el aula. Asimismo, de forma alternativa, entraban a las sesiones las dos alumnas invidentes con su acompañante, vivenciando la sesión dentro de la sala donde era llevada a cabo. Por último, a partir de la tercera sesión, se brindaba la oportunidad de que dos observadores-registradores voluntarios pudieran entrar a sesión por unos minutos como equipo reflexivo.

- 20:30h a 21:00h. Los/as alumnos/as realizaban una valoración personal de su experiencia como terapeuta-coterapeuta. Asimismo, se llevaba a cabo una puesta en común, haciendo reflexión grupal, compartiendo impresiones (dificultades encontradas, estrategias empleadas adecuadamente, estilo terapéutico), y aportando los docentes su feedback al grupo.

Evaluación final. Al finalizar la asignatura y con ello el programa de entrenamiento de HHSS, se administró de nuevo el cuestionario de evaluación y se solicitó a las alumnas invidentes la valoración sobre su experiencia en el grupo de terapia.

\subsection{Análisis de datos}

Para realizar el procedimiento estadístico se ha utilizado el programa SPSS 24.0. Las variables dependientes del estudio son de naturaleza cuantitativa, por ello, en primer lugar, hemos comprobado el supuesto de normalidad a través del cálculo de la significación de Kolmogorov-Smirnov en las variables de diferencia de las medidas test-retest para el rendimiento académico, las competencias específicas y la autoevaluación.

A partir de los resultados obtenidos, para los análisis intragrupo, cuando el supuesto de normalidad se cumple, es decir $\mathrm{p}>, 05$, se ha llevado a cabo un análisis de $t$ de Student para muestras relacionadas, teniendo en cuenta que una $\mathrm{p}<, 05$ indica diferencia significativa entre las medidas test-retest de las variables. De lo contrario, si el supuesto de normalidad no se cumple, hemos empleado una prueba no paramétrica, en este caso, rangos con signo de Wilcoxon, teniendo en cuenta que una $\mathrm{p}<, 05$ indica también una diferencia significativa entre las medidas test-retest. Además, calculamos el tamaño del efecto a través del índice $d$ (Cohen, 1988), teniendo en cuenta los siguientes puntos de corte: potencia estadística alta $(d= \pm 0,8)$, media $(d= \pm 0,5)$, baja $(d= \pm 0,2)$ y nula $(d=0)$.

Para los análisis intergrupos, si se cumple el supuesto de normalidad, es decir $\mathrm{p}>, 05$, hemos llevado a cabo un análisis de $t$ de Student para grupos independientes teniendo en cuenta que una $p<, 05$ indica diferencia significativa entre las puntuaciones medias de ambos grupos. Si, por el contrario, el supuesto de normalidad no se cumple $\mathrm{p}<, 05$, hemos realizado una prueba U de Mann-Whitney, teniendo en cuenta que una $\mathrm{p}<, 05$ indica una diferencia significativa entre las medianas de ambos grupos. Asimismo, calculamos el tamaño del efecto a través del índice $d$ (Cohen, 1988), teniendo en cuenta los puntos de corte señalados anteriormente.

\section{Resultados}

En primer lugar, con el análisis comparativo de las competencias específicas iniciales y finales, hemos encontrado que existen diferencias significativas entre las puntuaciones $(p<.05)$ y un tamaño del efecto alto en todas las variables $(\mathrm{d}>0.8)$. Es decir, se observa un aumento producido en las puntuaciones finales de cada competencia específica (Cuadro 1). 
Cuadro 1

Puntuaciones medias y pruebas para muestras relacionadas en las competencias especificas $(N=44)$

\begin{tabular}{|c|c|c|c|c|c|c|c|}
\hline & \multicolumn{2}{|c|}{$M(D T)$} & \multirow[t]{2}{*}{$t$} & \multirow[t]{2}{*}{$g l$} & \multirow[t]{2}{*}{$Z$} & \multirow{2}{*}{$\begin{array}{c}\text { Sig. } \\
\text { (bilateral) }\end{array}$} & \multirow[t]{2}{*}{$d$} \\
\hline & Inicial & Final & & & & & \\
\hline CE1 & $0,36(0,35)$ & $7,41(0,64)$ & 59,87 & 43 & &, $000^{*}$ & 13,68 \\
\hline CE2 & $0,19(0,16)$ & $0,96(0,11)$ & & & 5,80 &, $000^{*}$ & 5,61 \\
\hline CE3 & $0,02(0,07)$ & $0,97(0,10)$ & & & 6,07 &, $000^{*}$ & 11,01 \\
\hline CE4 & $0,15(0,21)$ & $3,06(0,46)$ & & & 5,80 &, $000^{*}$ & 8,14 \\
\hline CE9 & $0,27(0,26)$ & $4,08(0,47)$ & 43,93 & 43 & &, $000^{*}$ & 10,03 \\
\hline CE10 & $0,05(0,10)$ & $0,38(0,16)$ & & & 5,62 &, $000^{*}$ & 2,47 \\
\hline CE11 & $0,31(0,21)$ & $1,86(0,20)$ & & & 5,79 &, $000^{*}$ & 7,56 \\
\hline CE12 & $0,02(0,06)$ & $1,36(0,18)$ & & & 5,89 &, $000^{*}$ & 9,99 \\
\hline CE13 & $0,04(0,11)$ & $1,94(0,19)$ & & & 6,10 &, $000^{*}$ & 12,24 \\
\hline CE14 & $0,05(0,08)$ & $0,93(0,17)$ & & & 5,97 &, $000^{*}$ & 6,62 \\
\hline CE15 & $0,10(0,16)$ & $2,74(0,33)$ & & & 5,81 &, $000^{*}$ & 10,18 \\
\hline CE16 & $0,33(0,32)$ & $6,87(0,67)$ & 54,21 & 43 & &, $000^{*}$ & 12,46 \\
\hline CE18 & $0,30(0,29)$ & $4,95(0,57)$ & 42,53 & 43 & &, $000^{*}$ & 10,28 \\
\hline
\end{tabular}

Nota. * La puntuación es significativa en el nivel 0,05 (bilateral).

Además, encontramos que el 100\% de dichas competencias pasaron de "no superada" a "superada". Con un análisis de frecuencias más detallado de cada alumno/a, se ha observado que, de todas las competencias específicas evaluadas inicialmente, solo un 6,8\% (tres casos) superaron la CE2 "Aplicar los fundamentos de la bioética y el método de deliberación en la práctica profesional, ajustándose su ejercicio como profesional sanitario a lo dispuesto en la Ley 44/2003, de 21 de noviembre, de ordenación de las profesiones sanitarias"; otro 6,8\% (tres casos) superaron la CE10 "Saber comunicar y comunicarse con otros profesionales, $\mathrm{y}$ dominar las habilidades necesarias para el trabajo en equipo y en grupos multidisciplinares”; y un 2.3\% (un caso) superó la CE11“Conocimiento de las obligaciones y responsabilidades del personal sanitario relativas a la confidencialidad de la información y de la protección de datos personales de los pacientes".

Tras la puesta en marcha y finalización de esta metodología docente innovadora, todas las competencias específicas fueron superadas por el alumnado al finalizar el curso, a excepción de un $2.3 \%$ (un caso) que no superó la CE4, "Analizar críticamente y utilizar las fuentes de información clínica”; y un 15.9\% (siete casos), que no superaron la CE10, "Saber comunicar y comunicarse con otros profesionales, y dominar las habilidades necesarias para el trabajo en equipo y en grupos multidisciplinares".

Asimismo, se confirmó la existencia de diferencias significativas $(\mathrm{Z}=5,78, \mathrm{p}<, 000)$, a favor de las puntuaciones obtenidas por el alumnado en el cuestionario de evaluación final $(M=9,32$, DT $=0,76)$ frente al inicial $(M=0,50, D T=0,33)$, con una potencia del tamaño del efecto alto $(d=15,05)$. En función de las categorías establecidas para las puntuaciones del rendimiento académico global, observamos que las calificaciones medias pasan de "suspenso" a "sobresaliente".

Del mismo modo, con los datos obtenidos tras comparar las puntuaciones de la autoevaluación, (véase Cuadro 2) podemos afirmar con una diferencia significativa $(\mathrm{p}<, 05)$ el aumento de la autovaloración del alumnado al finalizar la asignatura. Además, en función de las categorías establecidas para las puntuaciones de la autoevaluación, observamos que el 100\% de las puntuaciones medias del alumnado pasan de "no superada" a "superada" con respecto a la asignatura y su ejercicio profesional en la autovaloración final sobre los conocimientos, habilidades/técnicas y responsabilidad. 
Cuadro 2

Prueba de rangos con signo de Wilcoxon para muestras relacionadas en la autoevaluación $(N=40)$

\begin{tabular}{lccccc}
\hline & \multicolumn{2}{c}{$\boldsymbol{M}(\boldsymbol{D T})$} & $\boldsymbol{Z}$ & Sig. & $\boldsymbol{d}$ \\
\cline { 2 - 6 } & \multicolumn{2}{c}{ Inicial } & Final & \multicolumn{3}{c}{ (bilateral) } \\
\cline { 2 - 6 } Conocimientos & $1,25(1,68)$ & $8,90(1,19)$ & 5,52 &, $000^{*}$ & 5,25 \\
Habilidades/Técnicas & $1,35(1,66)$ & $8,35(1,63)$ & 5,50 &, $000^{*}$ & 4,26 \\
Responsabilidad & $3,85(3,22)$ & $9,10(1,35)$ & 5,15 &, $000^{*}$ & 2,13 \\
Valoración global & $1,85(1,94)$ & $8,85(1,35)$ & 5,49 &, $000^{*}$ & 4,19 \\
\hline
\end{tabular}

Nota. ${ }^{*}$ La puntuación es significativa en el nivel 0,05 (bilateral).

Llevando a cabo un análisis de frecuencias detallado de cada alumno/a se ha observado que, en la autoevaluación inicial, solo un 4,9\% (dos casos) han obtenido una puntuación igual o superior a 5 en conocimientos. Asimismo, ningún caso ha superado las habilidades/técnicas. Sin embargo, respecto a la responsabilidad, se encontró un mayor número de calificaciones iguales o superiores a 5, siendo superada por un 42,5\% (17 casos). Tras la puesta en marcha y finalización de esta metodología docente innovadora, todos los alumnos/as obtuvieron una puntuación igual o superior a 5 en conocimientos, habilidades/ técnicas y responsabilidad. Solo 2,4\% (un caso) no superó las habilidades/técnicas. Finalmente, observamos que las puntuaciones medias del alumnado en la autovaloración global pasan de "suspenso" a "notable".

A continuación, se realizó una comparación entre las puntuaciones globales obtenidas en función del tipo de evaluación (evaluación docente y autoevaluación). Los resultados obtenidos confirman la existencia de diferencias significativas entre las puntuaciones, rechazando la hipótesis nula de igualdad de medianas $(\mathrm{Z}=3,79, \mathrm{p}<, 000)$ al inicio de la asignatura, con una potencia del tamaño de efecto alto $(\mathrm{d}=0,97)$. Igualmente, los resultados rechazan la hipótesis nula $(\mathrm{Z}=2,02, \mathrm{p}=, 043)$ en la comparación de las puntuaciones al final de la asignatura, pero con una potencia del tamaño de efecto bajo $(\mathrm{d}=0,43)$, lo que indicaba que el poder estadístico podría estar siendo excesivamente alto. Cuando lo reducimos a una $\mathrm{p}<, 01$, la diferencia significativa entre las puntuaciones de las evaluaciones iniciales se mantuvo, no resultando significativa esta diferencia en la evaluación final (error tipo I, falso positivo). Sin embargo, en función de la categoría establecida para las evaluaciones, se observa que alumnos/as y docentes coinciden en las puntuaciones iniciales "suspenso" y difieren en las puntuaciones finales, obteniendo el alumnado una puntuación media de "notable" mediante la autoevaluación y de "sobresaliente" a partir del rendimiento académico global. Además, se observa que en el inicio del curso las puntuaciones medias más altas corresponden a la autoevaluación global del alumnado. Sin embargo, al final de la asignatura, aunque ambas puntuaciones aumentan, la evaluación de los docentes es superior (véase Figura 1).

\section{Figura 1}

Puntuaciones medias según el tipo de evaluación, al inicio y final de la asignatura

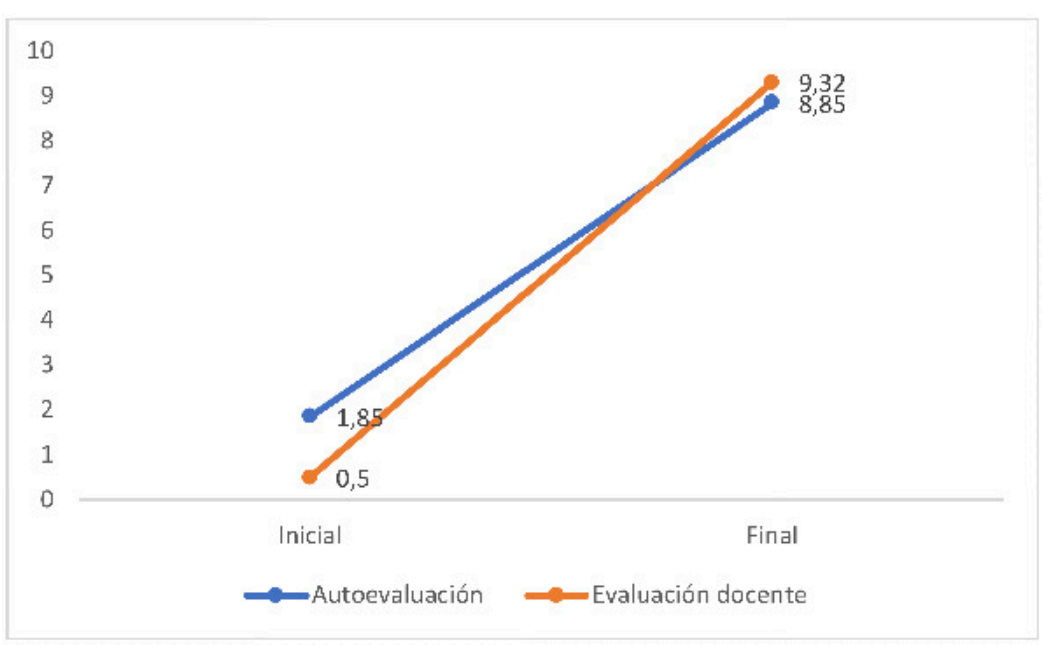

Nota. Elaboración propia. 
Por otro parte, en lo relativo a la comparación entre el grupo de participación directa (terapeuta-coterapeuta) y el grupo de participación indirecta (observador-registrador), hay que indicar que las puntuaciones analizadas corresponden con el resultado de la diferencia entre las puntuaciones test-retest para cada una de las variables estudiadas. Si observamos los resultados extraídos de estos análisis (véase Cuadro 3), podemos concluir que p >,05 indica que no se encontró diferencias significativas entre los dos grupos en ninguna de las 13 competencias específicas evaluadas. La potencia estadística observada también es baja-nula. Por lo tanto, podemos afirmar que no se han observado diferencias en la adquisición de las competencias específicas en función del tipo de participación del alumnado.

Con relación al rendimiento académico global en función del tipo de participación del alumnado, tampoco se han encontrado diferencias significativas entre los dos grupos $(\mathrm{Z}=1,50, \mathrm{p}=, 133)$. Las puntuaciones medias $(\mathrm{M}=8,90, \mathrm{DT}=0,87)$ obtenidas por el grupo de participación directa $(\mathrm{n}=29)$ son similares a las puntuaciones medías $(\mathrm{M}=8,65, \mathrm{DT}=0,77)$ del grupo de participación indirecta $(n=15)$. La potencia estadística observada también es baja $(d=0,30)$.

Cuadro 3

Puntuaciones medias y pruebas para muestras independientes en las competencias especificas

\begin{tabular}{|c|c|c|c|c|c|c|c|}
\hline \multirow[b]{3}{*}{ CE1 } & \multicolumn{2}{|c|}{$M(D T)$} & $t$ & $g l$ & $z$ & Sig. & $d$ \\
\hline & \multicolumn{4}{|c|}{$\begin{array}{c}\text { P.Directa }(n=29) \text { P.Indi- } \\
\text { recta }(n=15)\end{array}$} & \multicolumn{3}{|c|}{ (bilateral) } \\
\hline & $7,09(0,84)$ & $6,96(0,68)$ & 0,51 & 42 & & ,612 & 0,17 \\
\hline CE2 & $0,76(0,20)$ & $0,79(0,16)$ & & & $-0,30$ & ,763 & $-0,17$ \\
\hline CE3 & $0,95(0,12)$ & $0,94(0,10)$ & & & $-1,17$ & ,241 & 0,09 \\
\hline CE4 & $2,99(0,53)$ & $2,78(0,61)$ & & & $-1,09$ & ,276 & 0,37 \\
\hline CE9 & $3,86(0,55)$ & $3,70(0,63)$ & 0,87 & 42 & & ,392 & 0,27 \\
\hline CE10 & $0,36(0,18)$ & $0,28(0,22)$ & & & $-1,23$ & ,217 & 0,40 \\
\hline CE11 & $1,56(0,34)$ & $1,54(0,26)$ & & & $-0,55$ & ,583 & 0,07 \\
\hline CE12 & $1,34(0,17)$ & $1,33(0,17)$ & & & $-0,22$ & ,829 & 0,06 \\
\hline CE13 & $1,89(0,25)$ & $1,93(0,16)$ & & & $-0,05$ & ,961 & $-0,19$ \\
\hline CE14 & $0,88(0,19)$ & $0,87(0,24)$ & & & $-0,20$ & ,840 & 0,05 \\
\hline CE15 & $2,65(0,37)$ & $2,61(0,39)$ & & & $-0,39$ & ,695 & 0,11 \\
\hline CE16 & $6,60(0,85)$ & $6,43(0,71)$ & 0,67 & 42 & & ,507 & 0,22 \\
\hline CE18 & $4,71(0,71)$ & $4,52(0,76)$ & 0,79 & 42 & & ,436 & 0,26 \\
\hline
\end{tabular}

Nota. *La puntuación es significativa en el nivel 0.05 (bilateral).

Igualmente, no se han observado diferencias en la autoevaluación global o por competencias generales en función del tipo de participación del alumnado $(\mathrm{p}>, 05)$, siendo también la potencia estadística baja-nula (véase Cuadro 4). 
Cuadro 4

Prueba de U de Mann Whitney para muestras independientes en la autoevaluación

\begin{tabular}{|c|c|c|c|c|c|}
\hline \multirow[b]{3}{*}{ Conocimientos } & & & \multirow{3}{*}{$\begin{array}{c}\mathrm{Z} \\
-0,83\end{array}$} & \multirow{3}{*}{$\begin{array}{c}\begin{array}{c}\text { Sig. } \\
\text { (bilateral) }\end{array} \\
, .409\end{array}$} & \multirow{3}{*}{$\begin{array}{c}\mathbf{d} \\
0,05\end{array}$} \\
\hline & \multicolumn{2}{|c|}{$\begin{array}{c}\text { P.Directa }(\mathrm{n}=26) \text { P.Indirecta } \\
(\mathrm{n}=14)\end{array}$} & & & \\
\hline & $7,69(2,69)$ & $7,57(1,79)$ & & & \\
\hline Habilidades/Técnicas & $7,15(2,72)$ & $6,71(1,49)$ & $-1,25$ & ,213 & 0,20 \\
\hline Responsabilidad & $5,46(4,06)$ & $4,86(3,01)$ & $-0,90$ & ,368 & 0,17 \\
\hline Valoración global & $6,92(2,90)$ & $7,14(2,32)$ & $-0,03$ & ,977 & $-0,08$ \\
\hline
\end{tabular}

Nota: * La puntuación es significativa en el nivel 0.05 (bilateral).

Por último, se expone la valoración de las dos alumnas sobre las adaptaciones curriculares realizadas para su integración en las clases con sesiones de entrenamiento de HHSS para pacientes con trastorno psicológico y déficit en habilidades sociales. Por un lado, contaron con un acompañante que les informaba de lo que ocurría a nivel no verbal durante las sesiones tanto dentro de la sala como en el aula. Ambas expusieron que hay aspectos que necesitan que otra persona les describa, bien porque no pueden captarlos visualmente, o porque en ocasiones se unan varias voces y tengan dificultades para distinguir lo que necesitan escuchar. Además, fue importante que conocieran con anterioridad la sala y que en las primeras sesiones la persona acompañante les describiera en qué posición del círculo estaban sentado/a cada paciente. En posteriores sesiones ya reconocían a los pacientes por la voz. Asimismo, el espacio grupal previo y posterior a cada sesión refieren que les ayudó a consolidar el aprendizaje de lo vivido y contrastando su experiencia con los demás, afirmaron que se sintieron empoderadas al observar que no había muchas diferencias entre lo que ellas y otros alumnos/as habían percibido.

\section{Discusión y conclusiones}

En este estudio nuestro objetivo fue valorar la eficacia de una metodología de innovación docente propuesta e impartida en la asignatura "Salud en alteraciones mentales: propuesta para la intervención psicológica” del MPGS de la Universidad de Sevilla, durante los cursos 2017/2018 y 2018/2019. Esta metodología se basó en la participación del alumnado en el desarrollo de sesiones de entrenamiento de HHSS para pacientes con trastorno psicológico y déficit en habilidades sociales. Nuestros objetivos específicos consistieron en evaluar el efecto de desarrollar dicha intervención psicológica en el rendimiento académico global, la adquisición de competencias específicas propias del psicólogo/a general sanitario y la autoevaluación que realizaba el alumnado sobre su competencia con relación a la asignatura y a su profesión. Además, se tuvo en cuenta si se beneficiaron más, en estos términos, los estudiantes que participaron directamente en las sesiones (terapeutas-coterapeutas) frente a los que solo participaron como observadores-registradores. También se evaluó si existían diferencias entre las calificaciones y autoevaluaciones globales del alumnado. Asimismo, incorporamos a la experiencia a dos alumnas invidentes para valorar qué tipo de adaptaciones llevar a cabo.

En primer lugar, poniendo el énfasis en el aprendizaje por competencias, los resultados encontrados tras los análisis indican un aumento de las puntuaciones medias en las 13 competencias específicas relacionadas con la metodología innovadora. Es decir, el alumnado superó estas competencias a excepción de un caso $(2,3 \%)$ que no superó la CE4, "Analizar críticamente y utilizar las fuentes de información clínica”, y siete casos $(15,9 \%)$ que no superaron la CE10, "Saber comunicar y comunicarse con otros profesionales, y dominar las habilidades necesarias para el trabajo en equipo y en grupos multidisciplinares”. A pesar de alcanzar el objetivo propuesto, nos planteamos por qué una serie de alumnos/as no han superado estas dos competencias. Por un lado, se podrían revisar las preguntas que evalúan estas competencias. Por otro, pensando en la idiosincrasia de cada alumno, es posible que algunos factores como el estilo de afrontamiento o el uso de estrategias influyan en los resultados (Rodríguez, 2011; Tipismana, 2019). Consideramos que se pueden valorar mejoras en la metodología de innovación propuesta que faciliten la adquisición en todo el alumnado (por ejemplo, podríamos fomentar pequeños grupos de debate previos 
al intercambio posterior a cada sesión para que cada alumno/a pueda tener más oportunidades de aportar su opinión e intercambiar ideas).

Además, con relación al rendimiento académico global, los resultados también reflejan un aumento de las puntuaciones medias, observándose un cambio de "suspenso" a "sobresaliente". En este sentido, ambos hallazgos coinciden con los resultados alcanzados en otros estudios en relación con el uso de metodologías participativas (Benítez-Hernández et al., 2019; Rodríguez-Testal et al., 2009, 2017), confirmando que las metodologías didácticas basadas en la participación del alumnado en un contexto práctico profesional conllevan a una mejora de la formación. Como exponía Saul y otros (2016), aunque cada estudiante responde a un estilo particular y predominante de aprendizaje, si es estimulado por estrategias de enseñanza adecuadas, se fomentará la construcción de su propio conocimiento y con ello la mejora de su rendimiento. Por tanto, se avala la necesidad en el aula de metodologías participativas (Castañeda, 2016; Jenaro et al., 2016), otorgando un papel activo y reflexivo al alumnado, que fomente el aprendizaje autónomo pero guiado de los estudiantes (Fernández, 2010; Zabalza, 2013), a través del acercamiento al quehacer profesional (Delors, 1997; Valle y Manso, 2018; Villardón, 2006), y adaptando la formación universitaria a las exigencias del mundo laboral (Rodríguez, 2007).

Asimismo, hemos encontrado diversas referencias en la literatura que señalan esencial la autoevaluación en el proceso de valoración del aprendizaje, puesto que describen que la capacidad de valorar el propio progreso ya es parte de las competencias (Fernández, 2010; Gimeno y Gallego, 2007). En este sentido, los resultados obtenidos en nuestro estudio señalan que se produce un cambio respecto a la autoevaluación del alumnado, mostrándose un aumento de las puntuaciones medias en la valoración de su competencia tras las sesiones de entrenamiento de HHSS con pacientes con trastorno psicológico y déficit en habilidades sociales. En específico, todos los alumnos/as obtuvieron una puntuación igual o superior a 5 en conocimientos, habilidades/ técnicas y responsabilidad. Solo un caso (2.4\%) no superó las habilidades/ técnicas. También se obtuvo un aumento de las puntuaciones medias en la autoevaluación global, y las calificaciones pasaron de "suspenso" a "notable". Estos resultados plantean el beneficio de incorporar la autoevaluación en la universidad, algo que ya otros autores reflejaban en sus trabajos (Bronkbank y MacGill, 2002, Fernández, 2010; Gimeno y Gallego, 2007). Según Villardón (2006), la autoevaluación proporciona estrategias de desarrollo personal y profesional como la capacidad crítica, la autonomía, el compromiso, la responsabilidad y la motivación para el aprendizaje.

Por otro lado, al realizar una comparación entre el rendimiento global académico calificado por los docentes y las autoevaluaciones globales del alumnado, al inicio, se reflejó que existen diferencias estadísticamente significativas entre las mismas, aunque en ambos casos la media es de "suspenso". Sin embargo, con respecto a la evaluación final, aunque el análisis también apunta que existen diferencias significativas y observamos que las puntuaciones medias pasan de "notable" en las autoevaluaciones a "sobresaliente" en el rendimiento académico global, encontramos que la potencia estadística era baja, lo que nos llevó a señalar que podíamos estar ante un error de tipo I (falso positivo). A este respecto pensamos que si bien al inicio de la asignatura cuando el alumnado aún no se ha expuesto a las situaciones de aprendizaje puede ser normal encontrar diferencias entre ambas puntuaciones, con el diseño y desarrollo de las sesiones el alumnado puede observar qué necesita para afrontar las situaciones planteadas. Coincidiendo con Dochy y otros (1999), creemos que es en el transcurso de la asignatura cuando el alumnado va aprendiendo qué competencias debe asumir al respecto, lo que puede llevar a que las evaluaciones del aprendizaje realizadas por los docentes y el alumnado se aproximen. Además, la herramienta empleada para la evaluación no fue homogénea, por lo que esto también puede estar influyendo en los resultados obtenidos. En este sentido, otros autores no han encontrado diferencias significativas entre las puntuaciones del alumnado y del profesorado (Boud et al., 1995, como se citó en Fernández, 2010). No obstante, consideramos que no es tan relevante la exactitud entre las puntuaciones obtenidas es función del tipo de evaluación, como observar que la autoevaluación proporciona al alumnado una mejora de sus competencias.

Posteriormente, con relación al tipo de participación, los resultados indicaron que las diferencias entre ambos grupos no fueron significativas, es decir, no se halló que el grupo de alumnos/as que participó como terapeuta-coterapeuta obtuviera mayor rendimiento académico global, beneficios en la adquisición de competencias o hiciera una autoevaluación más alta, que el grupo que participó solo como observador-registrador. La falta de diferencias puede ser debida a que ambos grupos vivenciaron las sesiones, e incluso en algunos casos, miembros del grupo observador-registrador participaron como parte del equi- 
po reflexivo. La metodología propuesta trataba de involucrar a todo el grupo dotando al aprendizaje de funcionalidad, pese a que por la limitación de tiempo no era viable que todos participaran como terapeuta-coterapeuta en las sesiones. Asimismo, el espacio previo y posterior a las sesiones también invitaba a la expresión y reflexión grupal. Además, en todo momento eran acompañados y guiados por los docentes. Como algunos autores señalan, es necesario que la labor del docente no solo se limite a la transmisión de información y conocimientos, sino que se trata de que planteen situaciones al alumnado, que se acerquen a la realidad profesional, donde manifestándose alguna dificultad, los alumnos y alumnas pueda reflexionar y demostrar cómo hacen uso de sus conocimientos, habilidades y actitudes para establecer respuestas a la problemática (Delors, 1997; Villardón, 2006).

Respecto a las dos alumnas con discapacidad visual, se ha observado que esta metodología innovadora ha resultado beneficiosa para ambas, que afirmaban vivir y aprender de la experiencia como sus compañeros/as. Solo fueron necesario algunas adaptaciones como la ubicación dentro de la sala, la figura de un compañero y el uso de recursos verbales. Estas adaptaciones están en la línea de las recomendadas por otros autores citados (Abejón Mendoza et al., 2010; Castellana y Sala, 2005: Gross, 2014). Las alumnas informaron que el desarrollo de las sesiones y el espacio grupal previo y posterior a cada sesión les ayudó a consolidar el aprendizaje de lo vivido. Coincidiendo con Abejón Mendoza et al. (2010), esta retroalimentación permitió a todo el grupo establecer una interacción más dinámica y aprender de la experiencia. Así como señalan Adams y Holland (2006) y Moriña-Diez y otros (2013), una metodología didáctica basada en la participación beneficia la formación de todo el alumnado.

A pesar de todos nuestros esfuerzos esta investigación presenta algunas limitaciones. Por una parte, hubiera sido interesante comparar los resultados con un grupo control. Por otra, una mejora que puede incorporarse es someter el cuestionario de evaluación inicial y final a un proceso de validación. Esto podría indicar las fortalezas y dificultades de este sistema de evaluación. Sin embargo, es posible que, al ajustar el cuestionario a las diferentes competencias y tratar de abarcar gran parte de los contenidos de la materia, la consistencia interna del instrumento no sea adecuada. Sin embargo, es posible que un indicador de fiabilidad interjueces podría reforzar las consideraciones de este procedimiento de recogida de información. Asimismo, las competencias y valoraciones evaluadas por el alumnado y los docentes no fueron medidas mediante una prueba homogénea, siendo más específica en el segundo caso. Para futuras investigaciones, y basándonos en la literatura aportada, sería interesante que el alumnado tuviera la lista de competencias a alcanzar y se autoevaluaran a partir de la misma.

También consideramos que sería interesante seguir eliminando barreras permitiendo a todo el alumnado disfrutar de la experiencia de aprendizaje. Las adaptaciones curriculares llevadas a cabo nos han aportado un valor añadido al proyecto. Tras acabar el programa de entrenamiento de HHSS para pacientes con trastorno psicológico y déficit en habilidades sociales, queremos compartir que las dos alumnas informaron de algunas propuestas de mejora, como asistir a la preparación de las sesiones previo al desarrollo de las mismas, y en caso de participar como terapeutas, hacer dinámicas o role-playing con grupos reducidos de 2-3 personas, y contar con un coterapeuta durante las sesiones con el que puedan compartir sensaciones y les ayuden con los aspectos visuales.

En definitiva, la educación universitaria en España pasa por un proceso de transformación pedagógica inmerso en el marco del Espacio Europeo de Educación Superior (EESS). En este contexto, los resultados de nuestro estudio también avalan la necesidad de implementar más metodologías didácticas participativas e inclusivas, que relacionen el contenido de las asignaturas con el contexto profesional, fomentando un aprendizaje por competencias que permita a todo el alumnado tener un papel activo, reflexivo y más autónomo. Además, sería importante continuar trabajando en la mejora del sistema de evaluación del aprendizaje.

Por otro lado, en el ámbito sanitario, ya que la mejora de la atención a la salud mental pasa por la formación de los profesionales a cargo de su cuidado, este estudio cobra especial relevancia. Consideramos que esta experiencia de innovación ha permitido enriquecer el proceso de enseñanza-aprendizaje, motivado y práctico, alcanzando las competencias específicas propias del psicólogo/a general sanitario, lo que se espera mejore la atención clínica de estos profesionales.

A modo de conclusión, los resultados extraídos de esta experiencia de innovación se pueden concretar en función de los objetivos planteados. Por un lado, se ha producido un aumento de las puntuaciones 
medias de las 13 competencias específicas propia de la asignatura y desarrolladas a través de las sesiones. Las 13 competencias específicas, se consideran "superadas" en función de las puntuaciones medias alcanzadas por el grupo en cada una de estas. Igualmente, se ha producido un aumento de la puntuación media global del rendimiento académico del alumnado, que han pasado de "suspenso" a "sobresaliente". Asimismo, se ha producido un aumento de la puntuación del alumnado en la autoevaluación final sobre sus conocimientos, habilidades/técnicas y responsabilidad, que se consideran "superadas" en función de las puntuaciones medias alcanzadas. Asimismo, a nivel global, las puntuaciones pasan de "suspenso" a "notable".

Se han producido diferencias significativas entre las puntuaciones iniciales en función del tipo de evaluación, aunque coinciden en la clasificación de "suspenso". Sin embargo, al finalizar la asignatura, se observan diferencias significativas en las puntuaciones entre la autoevaluación global del alumnado "notable" y la evaluación global realizada por el profesorado "sobresaliente", pero con un bajo tamaño de efecto que nos indicaba que el poder estadístico $\mathrm{p}<.05$ estaba siendo alto. Cuando lo reducimos a una $\mathrm{p}<.01$, la diferencia significativa entre las puntuaciones de las evaluaciones iniciales se mantuvo, no resultando significativa esta diferencia entre las evaluaciones finales. Por otro lado, no se han encontrado diferencias significativas en la adquisición de competencias específicas, en el rendimiento global final o en la autoevaluación entre el alumnado que ha participado directamente (terapeutas-coterapeutas) o solo indirectamente (observadores-registradores).

\section{Referencias}

Abejón Mendoza, P., Martínez Solana, M. Y. y Terrón López, M. J. (2010). Propuesta de acción para la integración de universitarios con discapacidad visual y auditiva ante el reto de Bolonia. Revista de Docencia Universitaria, 8(2), 175-196. https://doi.org/10.4995/redu.2010.6202

Adams, M. y Holland, S. (2006). Inproving Access to higher education for disabled people. En M. Adams y S. Brown (Eds.), Towards inclusive learning in higher education (pp. 10-22). Routledge.

Álvarez, B., González, C. y García, N. (2007). La motivación y los métodos de evaluación como variables fundamentales para estimular el aprendizaje autónomo. Revista de Docencia Universitaria, 2, 1-12.

Armbruster, P., Patel, M., Johnson, E. y Weiss, M. (2009). Active learning and student-centered pedagogy improve student attitudes and performance in introductory Biology. CBE Life Science Education, 8(3), 203-213. https://doi.org/10.1187/cbe.09-03-0025

Benítez Hernández, M. M., Catalán Mahindo, M. C. y García Ocaña, C. (2019). Experiencia de innovación docente para el aprendizaje de competencias específicas del alumnado de la asignatura "Salud en alteraciones mentales: propuesta para la intervención psicológica”, del Máster de Psicología General Sanitaria (curso 2017/2018), a través del entrenamiento en habilidades sociales de pacientes con alteración mental [Simposio]. 7th International Congress of Educational Sciences and Development, Universidad de Granada.

BOE. (2013). Orden ECD/1070/2013, de 12 de junio, por la que se establecen los requisitos para la verificación de los títulos universitarios oficiales de Máster en Psicología General Sanitaria que habilite para el ejercicio de la profesión titulada y regulada de Psicólogo General Sanitario, núm. 142, del 14 de junio de 2013, (pp. 44948-44953). Ministerio de Educación, Cultura y Deporte.

Brockbank, A. y McGill, I. (2002). Aprendizaje reflexivo en la educación superior. Morata.

Castañeda, J. (2016). Análisis del desarrollo de los nuevos títulos de Grado basados en competencias y adaptados al Espacio Europeo de Educación Superior (EEES). REDU: Revista de Docencia Universitaria, 14(2), 135-157. https://doi.org/10.4995/redu.2016.5806

Castellana, M. y Sala, I. (2005). La universidad ante la diversidad en el aula. Aula Abierta, 85, 57-84. https://sid.usal.es/idocs/F8/ART9632/universidad_ante_diversidad_en_el_aula.pdf

Cohen, J. (1988). Statistical power analysis for the behavioural sciences. LEA. 
Colomo, E. y Gabarda, V. (2019). ¿Qué tipo de docentes tutorizan las prácticas de los futuros maestros de primaria? REICE. Revista Iberoamericana sobre Calidad, Eficacia y Cambio En Educación, 17(3), 59-78.

https://doi.org/10.15366/reice2019.17.3.004

De Flores, T., Lavilla, M., Molina, D. y López, B. (2007). Volver a aprender a montar en bicicleta. Morales i Torres.

Delors, J. (1997). La educación encierra un tesoro. UNESCO.

De Miguel, M. (2006). Modalidades de enseñanza centradas en el desarrollo de competencias. Orientaciones para promover el cambio metodológico en el espacio europeo de educación superior. Ediciones Universidad de Oviedo.

Dochy, F., Segers, M. y Sluijsmans, D. (1999). The use of self-, peer and co-assessment in higher education: A review. Studies in Higher Education, 24(3), 331-350. https://doi.org/10.1080/03075079912331379935

Fernández, A. (2010). La evaluación orientada al aprendizaje en un modelo de formación por competencias en la educación universitaria. REDU: Revista de Docencia Universitaria, 8(1), 11-34. https://doi.org/10.4995/redu.2010.6216

Fouad, N. A., Grus, C. L., Hatcher, R. L., Kaslow, N. J., Hutchings, P.S., Madson, M. B., Collins, F. L. y Crossman, R. E. (2009). Competency benchmarks: A developmental model for understanding and measuring competence in professional psychology cross training levels. Training and Education in Professional Psychology, 3, S5-S26. https://doi.org/10.1037/a0015832

García Cedeño, M.L., Maya Montalván, G.P., Pernas Álvarez, I.A., Bert Valdespino, J.E. y Juárez Ramos, V. (2016). La tutoría con enfoque inclusivo desde la universidad para estudiantes con discapacidad visual. Revista Cubana de Educación Superior, 35(3), 148-160. http://scielo.sld.cu/pdf/rces/v35n3/rces12316.pdf

García-Palacios, A., (2006). La terapia dialéctico comportamental. eduPsykhé: Revista de Psicología y Psicopedagogía, 5(2), 255-271.

Gimeno, M. y Gallego, S. (2007). La autoevaluación de las competencias básicas del estudiante de psicología. Revista de Psicodidáctica, 12(1), 7-28.

Gómez, E. (2007). Terapia dialéctica conductual. Revista de Neuro-Psiquiatria, 70(1-4), 63-69.

Gross, M. (2014). Prácticas inclusivas para la población estudiantil en condiciones de discapacidad visual en el entorno universitario. Alteridad. Revista de Educación, 9(2), 108-117. https://doi.org/10.17163.alt.v9n2.2014.02

Hatcher, R. L. y Lassiter, K. D. (2007). Initial training in professional psychology: The practicum competencies outline. Training and Education in Professional Psychology, 1, 49-65. https://doi.org/10.1037/1931-3918.1.1.49

Jenaro, C., Pérez, M. y Castaño, R. (2016). Reflexiones en torno a la adquisición de competencias a través del Máster Universitario en Psicología General Sanitario. [Comunicación]. EDUNOVATIC 2016 - I Congreso Virtual Internacional de Educación, Innovación y TIC, Madrid.

Lasa, A. (2017). Proyectos Docentes y de Investigación. UNED.

Linehan, M. M. (2003). Manual de tratamiento de los trastornos de personalidad límite. Paidós.

Moriña-Diez, A., López, R., Melero, N., Cortés, M. D. y Molina, V. M. (2013). El profesorado en la universidad ante el alumnado con discapacidad: ¿Tendiendo puentes o levantando muros? REDU: Revista de Docencia Universitaria, 11(3), 423-442.

Ramos-Villagrasa, P. J., Fernández-Campo, A., Oliván, B., Fernández del Río, E., Berges, A., Hernández, S., Huarte, S. y Martín-Peña, J. (2019). Storytelling: Una metodología de aprendizaje activo para la enseñanza de la psicología social en la educación superior. Summa Psicológica UST, 16(1), 11-19.

https://doi.org/10.18774/0719-448x.2019.16.1.349

Rodríguez, A. (2007). Las competencias en el Espacio Europeo de Educación Superior: Tipologías. Humanismo y Trabajo Social, 6, 139-153.

Rodríguez Ayán, M. N. y Ruíz Díaz, M. (2011). Indicadores de rendimiento de estudiantes universitarios: Calificaciones versus créditos acumulados. Revista de Educación, 355, 467-492.

Rodríguez Testal, J. F. (Coord.), Valdés Díaz, M., Benítez Hernández, M. M., García García, M., Blanco Gómez, J. L. y Senín Calderón, C. (2017). Material audiovisual para la enseñanza práctica en psicopatología clínica. Editorial Eos. 
Rodríguez Testal J. F. y León, J. L. (2002). Psicopatología clínica: Elaboración de un material didáctico. Revista de Enseñanza Universitaria, 19, 165-172.

Rodríguez Testal, J. F., Benítez Hernández, M. M. y Núñez Gaitán, M. C. (2009). Utilización de casos clínicos grabados en vídeo para la formación en psicopatología [Curso]. Encuentro sobre docencia de la facultad de psicología de la Universidad de Sevilla, Universidad de Sevilla.

Saúl, C., Rodríguez, G., Escoto, M. C. y Luna, E. (2016). Relación de los estilos y estrategias de aprendizaje con el rendimiento académico en estudiantes universitarios. Revista de Estilos de Aprendizaje, 9(17), 268-288.

Tejada, J. y Ruiz, C. (2016). Evaluación de competencias profesionales en educación superior: Retos e implicaciones. Educación XXI, 19(1), 17-38. https://doi.org/10.5944/educXXI.12175

Tipismana, O. (2019). Factores de resiliencia y afrontamiento como predictores del rendimiento académico de los estudiantes en universidades privadas. REICE. Revista Iberoamericana Sobre Calidad, Eficacia y Cambio en Educación, 17(2), 147-185. https://doi.org/10.15366/reice2019.17.2.008

UE, Unión Europea. (2008). Recomendación del parlamento europeo y del consejo relativa a la creación del marco europeo de cualificaciones para el aprendizaje permanente. Diario Oficial de la Unión Europea, 2008/C 111/01,1-7.

Valle, J. M. y Manso, J. (2018). El prácticum en la formación inicial: Aportaciones del modelo 9:20 de competencias docentes. Cuadernos de Pedagogía, 489, 33-40.

Villardón, L. (2006). Evaluación del aprendizaje para promover el desarrollo de competencias. Educatio Siglo XXI, $24,57-76$.

Zabalza, M. A. (2013). Innovación en la enseñanza universitaria. Contextos Educativos: Revista de Educación, 6-7, 113136. https://doi.org/10.18172/con.531

\section{Breve CV de los autores}

\section{Ma del Mar Benítez Hernández}

Profesora Contratada Doctora en el Departamento de Personalidad, Evaluación y Tratamiento Psicológicos.Facultad de Psicología (Universidad de Sevilla), imparte docencia en "Psicopatología Clínica” (Grado de Psicología) y en "Salud en alteraciones mentales: propuestas para la intervención” ( Máster de Psicología General Sanitaria). Ha colaborado en diferentes iniciativas relacionadas con la elaboración de material docente tales como Material Audiovisual para la enseñanza práctica en Psicopatología Clínica. Madrid. Ed. EOS.ISBN 978-84-9727-731-0 y Enseñando para la inclusión en la Universidad. De la teoría a la práctica. Ed. Narcea (en prensa). Así como su participación en el SIMPOSIO INVITADO: Innovaciones para la enseñanza en Psicología Clínica celebrado en el 7th International Congress of Educational Sciences and Development en 2019. Email: marbenitez@us.es

ORCID ID: https://orcid.org/0000-0001-8958-0344

\section{Juan Francisco Rodríguez Testal}

Doctor en Psicología, especialista en Psicoterapia, y profesor de Psicopatología de la Universidad de Sevilla (España). Tiene publicaciones, principalmente sobre psicosis, trastornos de la personalidad, envejecimiento, y alteraciones de la imagen corporal. Investigador que ha dirigido proyectos $\mathrm{I}+\mathrm{D}+\mathrm{I}$ y publicado monografías y manuales. Tiene publicaciones, principalmente sobre psicosis, trastornos de la personalidad, envejecimiento, y alteraciones de la imagen corporal. Es miembro del grupo de investigación Neurodesarrollo y psicosis del Instituto de Biomedicina de Sevilla (IBIS), y director del grupo de investigación CTS-301 Alteración Mental y Disfunción Social. Email: testal@us.es

ORCID ID: https://orcid.org/0000-0002-7346-5656 


\section{Macarena Torrado Herrera}

Máster en Psicología General Sanitaria por la Universidad de Sevilla (España). Ha sido miembro del departamento de Psicología Básica de la Universidad Pablo de Olavide (Sevilla, España) donde entre otros proyectos ha participado en investigaciones sobre dificultades del aprendizaje, funciones ejecutivas, envejecimiento, y ha colaborado en la adaptación y validación técnica de textos en Lectura Fácil. A nivel privado ha ejercido su labor en el ámbito del desarrollo y deterioro cognitivo, trabajando en la promoción y prevención de la salud, así como en la intervención con colectivos afectados por daño cerebral (enfermedad de Huntington, Demencias, Alzheimer, Parkinson, Ictus, Accidentes de tráfico). Email: psicologatorrado@gmail.com

ORCID ID: https://orcid.org/0000-0002-7918-4679 